\title{
Un Ciclo de Mejora en el Aula para materiales refractarios
}

\section{A Classroom Improvement Cycle for refractory materials}

DOMINGO MARTÍN GARCíA

Universidad de Sevilla

Dpto. Cristalografia, Mineralogía

y Química Agrícola

dmartin5@us.es

ORCID: https://orcid.org/0000-0003-3725-6598

Fecha de recepción del artículo: 31/05/19

Fecha de aceptación del artículo: 15/07/19

DOI: http://dx.doi.org/10.12795/9788447221912.049 Pp.: 1135-1151 


\section{Resumen:}

El presente Ciclo de Mejora en el Aula (CIMA) aborda el desarrollo los contenidos nucleares del tema de materiales refractarios de la asignatura de Materiales Cerámicos de los Grados de Ingeniera de Materiales y los Dobles Grados en Física e Ingeniería de Materiales y en Química e Ingeniera de Materiales. Centrados en cuatros preguntas principales se abordan estos contenidos interrelacionándolos y dirigiéndolos desde los primitivos de definición, propiedades, producción y seguimiento. El Ciclo se centra en el aprendizaje investigativo del propio estudiante apoyado bajo supervisión del docente. Ciclo que es evaluado para futuras mejoras.

Palabras claves: Materiales cerámicos, Ingeniera de Materiales, Doble Grado en Física e Ingeniería de Materiales, Doble Grado en Química e Ingeniería de Materiales, docencia universitaria, experimentación docente universitaria.

\section{Abstract:}

This Classroom Improvement Cycle (CIC) deals with the development of the nuclear contents of the subject of refractory materials of the course of Ceramic Materials of the Degrees of Materials Engineer and the Double Degrees of Physics and Materials Engineering, and Chemistry and Materials Engineer. Focused on four major questions, these contents are approached interrelating them and directing them from the original definition, properties, production and monitoring. The Cycle focuses on the student's own research learning supported under the supervision of the teacher. The Cycle is evaluated for future improvements.

Keywords: Ceramic materials, Materials Engineer, Double Degree in Physics and Materials Engineering, Double Degree in Chemistry and Materials Engineering, university teaching, university teaching experimentation. 


\section{Breve descripción del contexto}

La asignatura en la que se ha realizado este Ciclo de Mejora en el Aula (CIMA) es Materiales Cerámicos del tercer curso del Grado en Ingeniera de Materiales y de cuarto curso en los Dobles Grados en Física e Ing. de Materiales y en Química e Ing. de Materiales. La duración de CIMA ha sido de ocho horas divididas en cuatro sesiones de dos horas cada una. El tema para el cual se aplicó el CIMA fue sobre "Materiales Refractarios" del tercer bloque "Tecnología cerámica" de la asignatura.

La asignatura cuenta con unos 60 alumnos matriculados entre las tres titulaciones en las que se imparte -las tres titulaciones simultáneamente asisten a la asignatura-. Sin embargo, durante la aplicación del CIMA la media de asistencia fue inferior, unos 30-35 alumnos. Es de destacar que, al tratarse de una asignatura obligatoria en las tres titulaciones, estos alumnos tienen diferentes conocimientos previos y diferentes formas de trabajar en clase, diferenciándose patrones generales en función de su titulación.

Por último, remarcar que, aunque la aplicación del CIMA fue en el tema de Materiales Refractarios, con pocas modificaciones se podría aplicar fácilmente sobre otros temas del III Bloque de la asignatura como son los temas de Cerámica Estructural, Pavimentos y Revestimientos y Vitrocerámicas, dado que comparte patrones similares.

\section{Diseño previo del CIMA}

\section{Mapa de contenidos y secuencia de actividades}

El diseño está basado en cuatro contenidos nucleares que dan respuesta a cuatro preguntas fundamentales. Estos concentos nucleares, Definición-Clasificación, Propiedades, Criterios de diseño y Mecanismos de corrosión (Figura 1, Mapa de Contenidos) (Porlán, 2017). Estos 
contenidos nucleares están interrelacionados entre ellos mediante otros contenidos secundarios como son las Materias Primas, los Procesos de sinterización, y la distinción entre propiedades Mecánicas y Físico-Química.

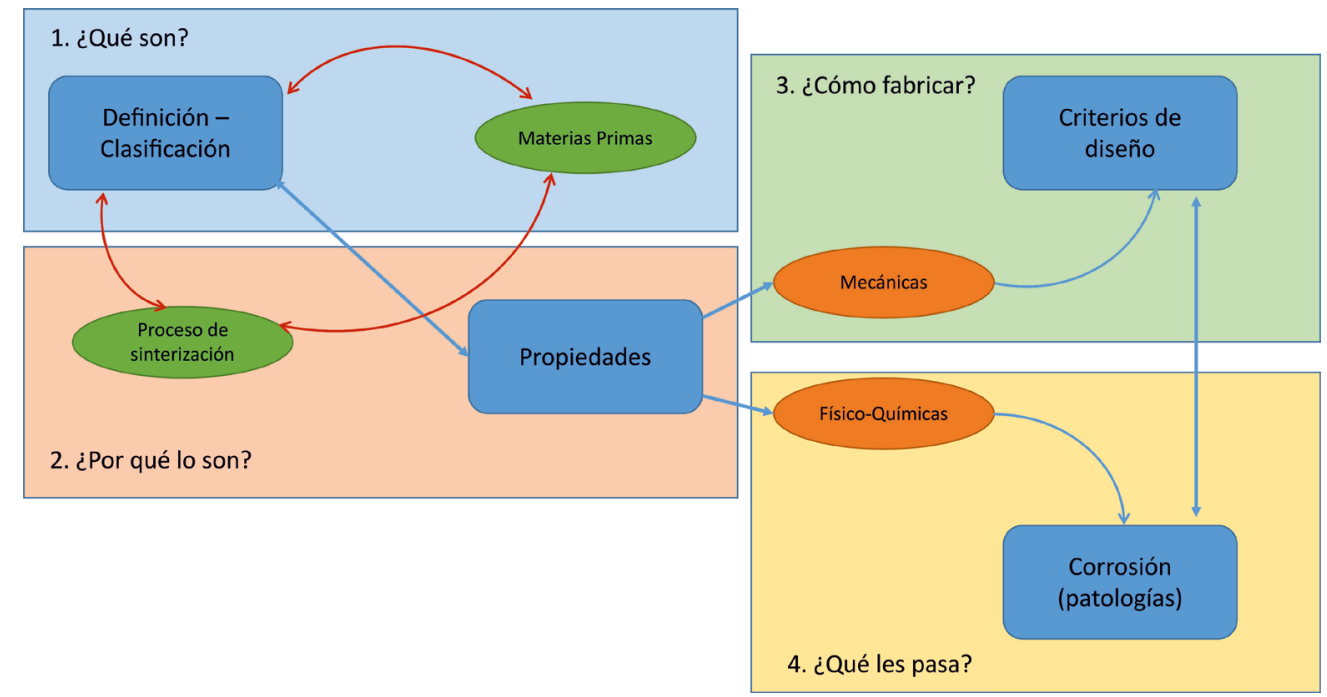

Figura 1. Mapa de contenidos del CIMA

El tema se ha dividido en cuatro preguntas principales que van desde la definición y clasificación de los refractarios respondiendo a la pregunta qué son, por qué lo son que vincula con las diferentes propiedades, pasando por cómo fabricarlos vinculando con los criterios de diseño, para terminar con qué les pasará en un futuro basándose en los mecanismos de corrosión.

Los contenidos abordados pueden clasificarse como conceptuales tales como la definición y clasificación, las propiedades mecánicas y físico-químicas como procedimentales, así como también lo son los criterios de diseño. A falta de contenidos actitudinales, que por norma general son poco comunes y olvidados en áreas de ciencia, serán tratados en el concepto de corrosión.

En la Figura 2 se recoge el modelo metodológico posible del CIMA. En él se ha distribuido el tiempo de manera 
que se dedique una cuarta parte (aproximadamente) a establecer el contenido puramente conceptual de definición clásica y las diferentes clasificaciones. Mientras que el tiempo restante, tres cuartas partes, se dedicarán a la práctica abordando los contenidos procedimentales relacionados con el análisis de resultados de medidas reales de propiedades y la metodología de fabricación.

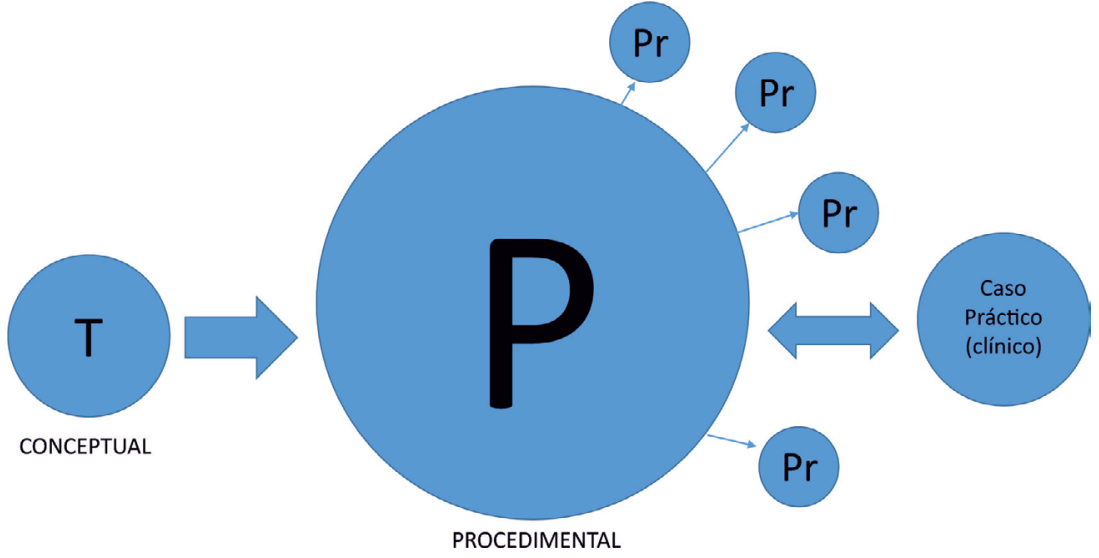

Figura 2. Modelo metodológico posible del CIMA. T: teoría; P: práctica; Pr: problema.

La secuencia de actividades están recogidas en la Tabla 1. Las actividades son tanto individuales como grupales con toda el aula o separados en diferentes grupos pequeños de 4 a 5 personas.

Tabla 1. Secuencia de actividades del CIMA.

\begin{tabular}{|c|c|}
\hline Concepto & Actividad \\
\hline $\begin{array}{l}\text { Definición y } \\
\text { clasificación ( } 2 \\
\text { horas) }\end{array}$ & $\begin{array}{l}\text { - Definición clásica de refractarios. } \\
\text { Clasificación. En lugar de dar los listados de clasifi- } \\
\text { cación, relacionar con las materias primas y con los } \\
\text { procesos de sinterización, preguntar cómo los cla- } \\
\text { sificarían según esos criterios. Completar y corregir } \\
\text { con las clasificaciones reales. }\end{array}$ \\
\hline
\end{tabular}

Jornadas de Formación e Innovación Docente del Profesorado | № 2 (2019) Esta obra se distribuye con la licencia Creative Commons Reconocimiento-NoComercial-SinObraDerivada 


\begin{tabular}{|c|c|}
\hline Propiedades (1:30) & $\begin{array}{l}\text { - Repaso rápido de las propiedades (ya las conocen } \\
\text { de otros temas y cursos). } \\
\text { - Dar ejercicios para determinar la propiedad a par- } \\
\text { tir de datos reales de medidas en equipos (medidas } \\
\text { indirectas), tanto de los refractarios como de otros } \\
\text { materiales cerámicos. } \\
\text { - En función de los resultados anteriores, hacer una } \\
\text { búsqueda en las normas UNE, si esos datos corres- } \\
\text { ponden a un refractario y de qué tipo. }\end{array}$ \\
\hline $\begin{array}{l}\text { Criterios de diseño } \\
\text { (1 hora) }\end{array}$ & $\begin{array}{l}\text { Visualizar video de YouTube sobre fabricación de la- } \\
\text { drillos refractarios. https://youtu.be/crxnKijsPas } \\
\text { - Ir interrumpiendo el vídeo para hacer preguntas so- } \\
\text { bre lo que acaban de ver. Tipo ¿por qué ese mez- } \\
\text { clado? ¿para qué es necesarios el aditivo? ¿por qué } \\
\text { es importante el secado previo a la sinterización? } \\
\text { ¿por qué ese método de prensado y no otro? ¿ven- } \\
\text { tajas de la sinterización utilizada? ¿por qué sinteri- } \\
\text { zación en etapas?... }\end{array}$ \\
\hline $\begin{array}{l}\text { Mecanismos de } \\
\text { corrosión (3:30) }\end{array}$ & $\begin{array}{l}\text { Proponer a los alumnos en grupos de no más de } 5 \\
\text { personas que resuelvan un "caso clínico" de corro- } \\
\text { sión de refractarios. Dando un diagnóstico, una cura } \\
\text { y prevención para futuros refractarios. Entregar un } \\
\text { informe breve. }\end{array}$ \\
\hline
\end{tabular}

\section{Cuestionario inicial y final para evaluar el conocimiento de los estudiantes}

El cuestionario propuesto pretende recoger la evaluación de ideas de los estudiantes tanto antes y después de la aplicación del CIMA. Los cuestionarios fueron pasados en clase, el inicial unos días antes de la sesión de aplicación y el final en la última sesión. Se realizaron en papel y en el momento indicado para que fuera contestado desde el conocimiento propio y no en la búsqueda de la solución correcta en cuestión. Fueron cuestionarios anónimos, pero con una codificación de tres cifras numéricas asignadas al 
azar que tendrán que recordar los alumnos para indicar el mismo código en el final y así poder comparar las respuestas antes y después de la aplicación. El cuestionario fue el siguiente:

Con este cuestionario anónimo (pero numerado al azar*) sólo se pretende conocer algunas actividades e ideas sobre aspectos fundamentales relacionados con el tema Cerámicas Refractarias.

1. ¿Qué entiendes (o sabes) por material refractario?

2. Defina el término de arcilla refractaria

3. Para el estudio de un material refractario, qué propiedades le parecen más interesantes.

4. ¿Qué técnicas utilizaría para caracterizar un material refractario?

5. Indique los campos de aplicación que cree que tiene los materiales refractarios.

¡Gracias por su colaboración!

*Por favor, recuerde el número del cuestionario para el cuestionario final

\section{Aplicación del CIMA}

\section{Relato resumido de las sesiones}

Las sesiones de aplicación del CIMA se trabajaron de forma satisfactoria como sensación general. El CIMA fue aplicado en cuatro sesiones de 2 horas cada una (realmente son de 1:50 dado que así lo establece el horario de clases en el centro, para que haya 10 minutos entre clases). Procedo a relatar cómo fueron cada una de estas sesiones:

\section{Sesión 1:}

Esta sesión fue dedicada por completo a los contenidos conceptuales de Definición y Clasificación de los 
materiales refractarios, relacionándolos de forma cíclica con los contenidos secundarios de Materias primas y Procesos de sinterización. Para la definición se partió de la definición clásica de cerámica refractaria. Desde este punto nos fuimos moviendo a contenidos secundarios, relacionándolos entre ellos. Para las clasificaciones, en lugar de dar los diferentes listados de clasificación, se les preguntó a los estudiantes que hicieran una clasificación en función de las principales materias primas y los estados de sinterización. Esto se trabajó en el aula todos juntos. Una vez establecidos unas clasificaciones en base a esos criterios se les dirigió y corrigió sus ideas de clasificación hasta llevarlos a las clasificaciones reales en base a materias primas y sinterización. Finalmente, se les mostró las demás clasificaciones habituales de los refractarios.

Al final de la sesión, quedó tiempo a introducir algunas propiedades que estaban relacionadas con las clasificaciones.

En general, las sensaciones al finalizar la sesión fueron muy satisfactorias. Sin embargo, en el momento de hacer partícipe a los estudiantes de clase, cuando tuvieron que proponer clasificaciones no hubo una respuesta inmediata, pero tras ofrecerles algunas ideas y pistas comenzaron a surgir nuevas ideas por parte de los ellos y a crear relaciones. La mayor satisfacción procede de que los estudiantes comienzan a enlazar conceptos básicos desde el comienzo.

El aspecto negativo es la falta de respuesta en los primeros minutos, habiendo momentos de silencio y la necesaria búsqueda de la participación.

\section{Sesión 2:}

Se continua con las propiedades, retomando desde donde se dejó en la sesión anterior, pero a la vez recordando 
lo dicho. Realmente, todo fue más un repaso de diferentes propiedades y magnitudes ya que eran conocedores de ellas de otras asignaturas y cursos.

La mayor parte del tiempo programado fue dedicado a resolver problemas relacionados con la medida real de propiedades de materiales cerámicos refractarios y no refractarios. Con esos datos, los estudiantes individualmente tuvieron que buscar en las normas UNE, habiéndoles dado previamente indicaciones para hacer la búsqueda de las normas y como deben ser interpretadas, para establecer cuáles de los materiales a los que se les había calculado esas propiedades podría ser considerado refractario y cuáles no.

En lo positivo, las sensaciones fueron bastante satisfactoria al tratarse de una sesión muy práctica y que los estudiantes están dispuestos a trabajar manejando datos fácilmente. En lo negativo, fue que no dio tiempo a tener analizados y establecido el uso como refractario durante la sesión, así que se le instó a realizar un pequeño informe que podrían entregar en la sesión siguiente.

\section{Sesión 3:}

En esta sesión se trabajó sobre los procesos de fabricación de materiales refractarios. Para ellos se utilizó un video de YouTube sobre la fabricación de ladrillos refractarios. Lo previsto fue poner el video e ir haciendo paradas (programadas) en las que hacerles preguntas y relacionar conceptos. Sin embargo, a pesar de comprobar previamente el video se podía reproducir, no se comprobó que el sonido fuera bueno, de modo que gran parte del tiempo entre paradas tenía que repetir que se había dicho en el video. Por tanto, se alargó más de los previsto.

En la segunda parte de la sesión estaba previsto la presentación y comienzo de los casos prácticos relacionados 
con los mecanismos de corrosión. Por falta de tiempo, solo se pudo indicar qué se quería hacer, cómo debían de trabajar, etc. Así como también se le dedico un tiempo considerable para que se dividieran en grupos de 4-5 personas y en distribuir los diferentes "casos clínicos" a resolver.

Por tanto, las sensaciones fueron menos satisfactoria debido a los inconvenientes relacionados con el sonido del video. Además, es necesario incentivar a los estudiantes al trabajo en grupo y enseñarles a hacerlo de forma eficiente. Son incapaces hasta de dividirse en grupo de forma rápida, siendo algunos de ellos tan pasivos que ni siquiera se distribuyen. Este último aspecto debe ser trabajado de otra forma o establecido previamente bajo algún tipo de criterio para la formación de los grupos.

\section{Sesión 4:}

La última sesión de aplicación del CIMA se dedicó a la realización por parte de los estudiantes en grupos de los "casos clínicos de patología" de los materiales refractarios, en definitiva, en analizar el proceso de corrosión que pueden darse en estos materiales. En todo momento este trabajo fue supervisado, orientando, ayudando y corrigiendo en el momento.

Al final de la clase se les pidió a los grupos que redactasen un breve informe en los que tendrían que:

- Identificar el tipo de corrosión, "diagnosticar la patología"

- Establecer el mecanismo de corrosión, "la cadena epidemiológica"

- Revolver la corrosión, "tratamiento y curación"

- Prevenir la aparición del tipo de corrosión, "prevención".

Esta actividad fue muy satisfactoria, creo que realmente ha funcionado. Fue novedosa y bien recibida por los estudiantes. 
Al final de esta sesión se pasó de nuevo el cuestionario.

En general, la sensación de la aplicación del CIMA fue satisfactoria. Ayuda mucho salir de lo tradicional a pesar del trabajo que requiere salir de la zona de confort. Requiere de trabajo previo, como en mi caso en la búsqueda de diferentes tipos de corrosión o de datos reales de medidas de propiedades de cerámicos. Por último, no tuve gran capacidad de modificar en el último momento el CIMA del modelo ya establecido antes de su aplicación, lo mantuve inamovible.

\section{Evaluación del aprendizaje de los estudiantes}

Para la evaluación del aprendizaje de los estudiantes se ha realizado mediante la comparación de las respuestas del cuestionario pasado antes y después de la aplicación del CIMA.

Este cuestionario basado en cinco preguntas básicas relacionadas con los contenidos nucleares tratados, fueron respondidos al inicio (pre) por 14 estudiantes y al final del ciclo (post) se obtuvieron 37 respuestas entre los que se incluyen los 14 que respondieron al inicio. De modo, que sean utilizado los 14 cuestionarios que se han completado en ambas etapas para la evaluación, sin embargo, se hará un análisis extrapolado teniendo en cuenta las 23 respuestas restantes.

Las respuestas a cada pregunta se clasifican en $5 \mathrm{ni}$ veles en función del nivel complejidad de la respuesta, siendo: 1- sin respuesta o incorrecta; 2 - respuesta que es considerada correcta pero muy básica; 3- respuesta correcta pero concisa, sin detalles; 4- correcta y detallada; 5- correcta y con interrelaciones con otros conceptos, respuesta de nivel avanzado.

Jornadas de Formación e Innovación Docente del Profesorado | № 2 (2019) Esta obra se distribuye con la licencia Creative Commons 
El análisis de evaluación del aprendizaje basado en los cuestionarios pre y post aplicación del CIMA es recogido en la Figura 3, de la cual se puede extraer las siguientes conclusiones de evaluación:

- El general, el CIMA ha ayudado a que las ideas de la mayoría de los estudiantes pasen de desconocimiento a nivel medios-avanzados.

- La primera pregunta, la más general de todas, los avances son claros, pero estando muy centrados en los niveles intermedios.

- La segunda pregunta, los avances están más distribuido de forma equitativa en todos los niveles, salvo el nivel más bajo, de donde partían inicialmente, en los que no se quedó ningún estudiante.

- Las preguntas relacionadas con técnicas y propiedades, las cuales en el test inicial presentaban respuestas con un nivel medio-bajo, son en las que se han conseguido subir a niveles más avanzados, lo cual demuestra un interés por parte del estudiante más importante frente a cuestiones más conceptuales.

P1: Pre: 0\%; Post: 7,1\% P2: Pre: 0\%; Post: 21,4\% P3: Pre: $0 \%$; Post: $28,6 \%$ P4: Pre: $0 \%$; Post: $21,4 \%$

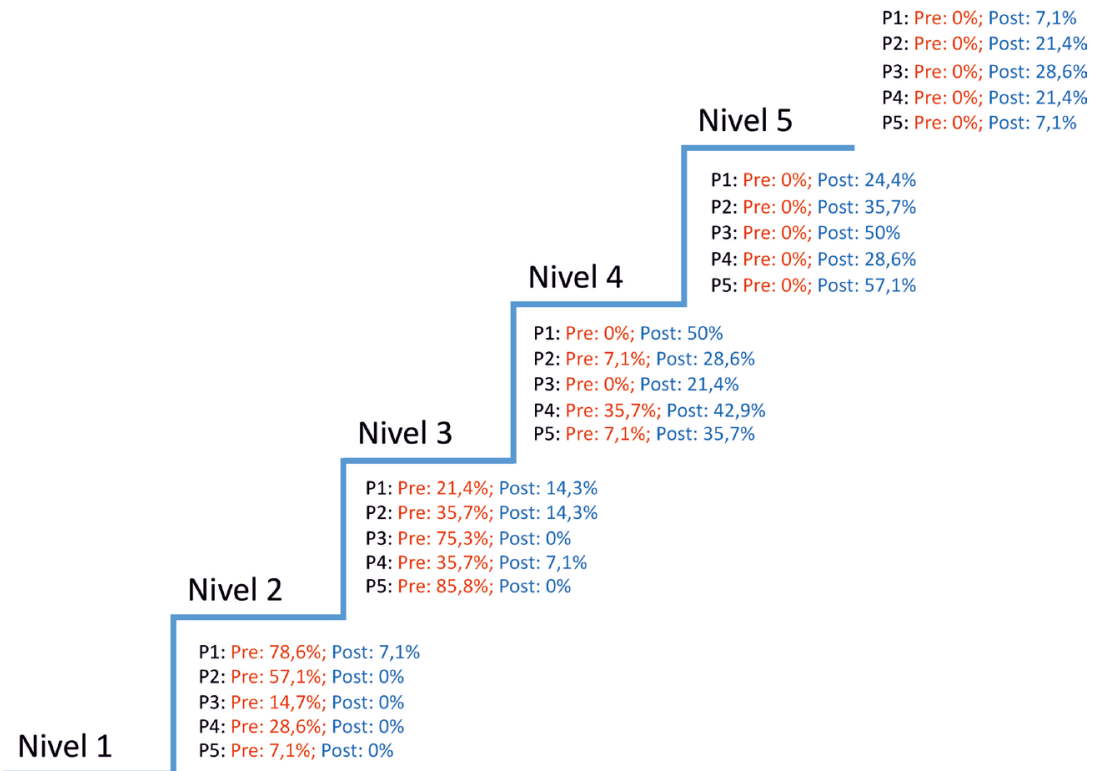

Figura 3. Evaluación mediante escaleras del aprendizaje de los alumnos. 
Sin embargo, como crítica el procedimiento empleado, las respuestas de los alumnos fueron inmediatas, al pasar el cuestionario al final de la última sesión de aplicación del ciclo, sin darles tiempo a la asimilación completa de la información. Se optó a hacerlo de esta forma, como ya se ha comentado, para evitar que los estudiantes buscasen las respuestas en lugar de responder en base a los conocimientos adquiridos.

\section{Evaluación del CIMA}

\section{Cuestiones a mantener y cambios a introducir para un futuro CIMA}

En líneas generales me encuentro satisfecho con los resultados obtenidos tras la aplicación del CIMA. Se considera que gran parte de los estudiantes han adquirido mediante este aprendizaje un conocimiento avanzado o al menos suficiente. Además, los estudiantes han mostrado un interés en la participación en las actividades planteadas mayor que con metodologías tradicionales.

La forma de trabajar los contenidos con mapas y problemas o actividades es efectiva para facilitar el aprendizaje de los estudiantes. Esta metodología de trabajo hace que tengamos una guía de los que queremos enseñar de una forma clara, ordenada y estructurada, mejorando la práctica docente. Mientras que a los estudiantes estos mapas les pueden ayudar a estructurar sus esquemas mentales sobre los contenidos tratados.

La utilización de cuestionarios para la evaluación de los conocimientos previos y finales de los estudiantes son una herramienta muy útil. Especialmente, considero que el análisis de las respuestas de los cuestionarios previos 
ayuda a modelar la metodología docente para el tema a tratar. Por ejemplo, en la aplicación del presente CIMA los conceptos relacionados con las propiedades fueron trabajos de forma diferentes, en el pasado eran tratados principalmente como conceptuales con los cuales se realizaban algunos ejercicios de cálculo y se tomó la decisión de realizar una actividad más centrado en los ejercicios y mucho menos en conceptos ya que estos conceptos eran conocidos por los estudiantes previamente.

Junto con la programación de actividades atractivos para atraer la atención de los estudiantes, las dos herramientas anteriores son consideradas las principales a mantener en el futuro.

En cuanto a cambios a introducir, las preguntas utilizadas en los cuestionarios no eran complemente apropiadas no por su contenido sino en la forma porque eran muy genéricas, especialmente las ultimas. Esto hace que sea complejo clasificar las respuestas en nivel. Aunque la programación de actividades ha sido positiva, haría cambios de algunas actividades. Ejemplo sería la actividad de vídeo, no solo por los problemas de la reproducción, sino más bien por el tipo de video empleado, pero existe el inconveniente al menos para este tema no disponer de mucho material.

\section{Aspectos de la experiencia que se pretenden incorporar a toda la práctica docente habitual}

La incorporación de los mapas de contenidos por temas o bloques temáticos y los cuestionarios, especialmente los previos, son considerados importantes a añadir a la práctica docente. La importancia de conocer los conocimientos previos de los estudiantes para moldear la secuencia de actividades en consecuencia es de gran utilidad, conocer en qué nivel o escalón de la escalera de 
conocimientos para ir moviendo hasta niveles de mayor complejidad.

Otro aspecto a considerar es necesidad de implicar al estudiante en su proceso de aprendizaje, favoreciendo y centrando este proceso de aprendizaje en ellos mismos (Bain, 2006). Favorecer un ambiente crítico en el aula haciendo que estos se enfrenten a tareas que deben resolver por sí mismo, aunque estén guiados por un docente (Finkel, 2008).

Los trabajos en grupos, en ellos se favorece que se expresen ideas uno a los otros a la hora de resolver problemas o actividades, buscando o presentado nuevas ideas y escuchándose entre ellos.

\section{Principios Didácticos que han guiado la experiencia}

Los Principios Didácticos que han guido la experiencia son la utilización de mapas de contenido con un número apropiado de conceptos nucleares y secundarios donde esta jerarquizada previamente la metodología de enseñanza. El uso de preguntas o actividades permite discriminar lo que realmente es importante y necesario para el aprendizaje adecuado.

Atraer la atención del estudiante al aprendizaje mediantes actividades que les comprometan en su propio aprendizaje. Es esencial en este aspecto que hay actividades en grupo que impliquen un compromiso compartidos entre los miembros del grupo.

Es necesario conocer los modelos mentales de cada uno de los estudiantes antes de abordar los contenidos. Para ello, es de gran utilidad el uso de cuestionarios bien diseñados y que permitan un análisis individualizado de cada estudiante y a su vez del conjunto de todos ellos. 
Por úlitmo, y de gran importancia, es la evaluación continua del modelo metodológico empelado para conocer los puntos débiles para mejorarlo y permitiendo modificar la secuencia de actividad diseñados previamente si fuera necesario para facilitar el aprendizaje.

Jornadas de Formación e Innovación Docente del Profesorado | № 2 (2019) Esta obra se distribuye con la licencia Creative Commons 


\section{Referencias bibliográficas}

Bain, K. (2006). Lo que hacen los mejores profesores de universidad. Valencia: Universitat de València.

Finkel, D. (2008). Dar clase con la boca cerrada. Valencia: Universitat de València.

Porlán, R. (coord.) (2017). Enseñanza Universitaria. Cómo mejorarla. Barcelona: Editorial Morata.

Jornadas de Formación e Innovación Docente del Profesorado | № 2 (2019) Esta obra se distribuye con la licencia Creative Commons 